\title{
Application of Boyd's Periodization and Relaxation Method in a Spectral Atmospheric Limited-Area Model. Part II: Accuracy Analysis and Detailed Study of the Operational Impact
}

\author{
DAAn Degrauwe \\ Royal Meteorological Institute, Brussels, Belgium \\ STEVEN CALUWAERTS \\ Department of Physics and Astronomy, Ghent University, Ghent, Belgium \\ FABRICE VOITUS \\ CNRM, Météo-France, Toulouse, France \\ RAFIQ HAMDI \\ Royal Meteorological Institute, Brussels, Belgium \\ PIET TERMONIA \\ Royal Meteorological Institute, Brussels, and Department of Physics and Astronomy, Ghent University, Ghent, Belgium
}

(Manuscript received 31 January 2012, in final form 6 April 2012)

\begin{abstract}
Spectral limited-area models face a particular challenge at their lateral boundaries: the fields need to be made periodic. Boyd proposed a windowing-based method to improve the periodization and relaxation. In a companion paper, the implementation of this windowing method in the operational semi-implicit semiLagrangian spectral HARMONIE system was described and some first reproducibility tests, comparing this method to the old existing one, were presented.

The present paper provides an in-depth study of the impact of this method for different configurations of the implementation. This is carried out in three steps in well-controlled experimental setups of increasing complexity. First, different aspects of Boyd's method are analyzed in an idealized perfect-model test using a representative 1D shallow-water model. Second, the implementation is tested in an adiabatic 3D numerical weather prediction (NWP) model with perfect-model experiments. Finally, the impact of using Boyd's method in a more operational-like NWP context is investigated as well. The presented tests show that, while the implementation of Boyd's method is neutral in terms of scores, it is superior to the existing spline method in the case of strong dynamical forcings at the lateral boundaries.
\end{abstract}

\section{Introduction}

Limited-area models (LAMs) have become a popular tool for short-range numerical weather prediction and for regional climate studies. Since they are running on smaller domains than global models, they can provide applications of higher resolution for comparable

Corresponding author address: Daan Degrauwe, Royal Meteorological Institute, Ringlaan 3, B-1180 Brussels, Belgium. E-mail: daan.degrauwe@meteo.be computing costs allowing one to simulate finescale phenomena more accurately. Developing and maintaining an operational LAM poses a few extra difficulties with respect to global models. Most importantly, the lateral boundary conditions (LBCs) need to be specified at the domain boundaries. This requires a stable numerical formulation of the LBCs and second that the data of the fields on the lateral boundaries are provided from another numerical weather prediction (NWP) model running at a lower resolution on a larger domain. 
In some atmospheric models, such as the Integrated Forecast System (IFS) of the European Centre for Medium Range Weather Forecasts (ECMWF) and the Action de Recherche Petite Echelle Grande Echelle (ARPEGE) model of Météo-France, the derivatives are computed in spectral space. However, it is well known that spectral transforms can only be performed on fields that are periodic. Haugen and Machenhauer (1993) proposed a method that allows for the development of spectral LAMs by introducing an artificial extension zone to the domain where the fields are made periodic. This technique has been successfully applied to develop operational numerical weather prediction models such as the Aire Limitée Adaptation Dynamique Développement International (ALADIN) model (ALADIN International Team 1997) and the HighResolution Limited-Area Model (HIRLAM) model.

Boyd (2005) proposed a windowing-based method for the periodization and relaxation of LAM fields. Termonia et al. (2012, hereafter Part I) have implemented this new method in the HIRLAM-ALADIN Research on Mesoscale Operational NWP in Euro-Mediterranean Partnership (Euromed) (HARMONIE) system, which comprises the ALADIN model, the ALARO model, and the Applications of Research in Operations at Mesoscale (AROME) model, see that paper for a detailed description of the model versions. The dynamical core of this system is a two-time-level semi-implicit semiLagrangian system that is different from the numerical scheme applied in Boyd (2005) and thus his proposal cannot be implemented straightforwardly without considering these differences first. Part I also presented a first reproducibility test comparing Boyd's method and the method used in the ALARO model configuration of the so-called HARMONIE system. This shows that the application of Boyd's method is feasible in an operational context and that it does not significantly affect the forecast scores; in other words, it is safe to replace the old existing method by a windowing-based method without loss of forecast skills. However, given the theoretical superiority of Boyd's method with respect to the existing spline method, one concludes either (i) that the errors due to periodization and relaxation are quite small in comparison with other model errors and observation errors, or (ii) that the improvements only occur in rare but extreme cases of strong dynamical forcings at the lateral boundaries. A well-controlled experimental approach is needed to investigate this.

In this paper, the superiority of Boyd's method in terms of accuracy will be demonstrated using a perfectmodel experiment setup. First, a detailed analysis of different aspects of Boyd's method on a semi-implicit semi-Lagrangian spectral LAM will be carried out using highly idealized models where other model errors are avoided as much as possible. Later, the step toward more operational-like situations is made. To this end we rely on perfect-model tests to avoid drawing erroneous conclusions due to limitations in the observations. The realistic tests will consists of adiabatic tests and tests where the model is run with the physics parameterization switched on.

In the present paper, we do not repeat the explanation of the implementation of the windowing method within the HARMONIE system. Instead, we refer the reader who is not familiar with this to Part I.

\section{Analysis}

A few aspects described in Part I are investigated in detail in this section. There are several aspects about the difference between Boyd's windowing-based method for periodization and relaxation and the spline-based method currently used in the HARMONIE system. First, Boyd's method delivers fields that are infinitely differentiable across the borders of the physical domain, whereas the spline-based method only yields secondorder continuity. Second, Boyd proposes to construct the fields in the extension zone using information from the large-scale model, whereas the content of the extension zone generated by the spline-based method is purely artificial. Third, Boyd proposes to use the same windowingbased strategy for periodization and for relaxation.

We will use the same classification of the experiments as in Part I, where an experiment is characterized by three or four letters:

- periodization with splines (S) or with Boyd's windowingbased method (B);

- relaxation with the existing ALADIN function (A) or with the erf-based function (E);

- overlapping $(\mathrm{O})$ or separate $(\mathrm{S})$ relaxation and periodization zones;

- truncation of semi-Lagrangian trajectories at the boundary of the domain $(\mathrm{T})$ or no truncation $(\mathrm{N})$. This option is only relevant in case of separate relaxation and periodization zones.

For instance, the current configuration of the HARMONIE system is denoted SAST, whereas Boyd proposes to use BEO.

Each of these aspects will be investigated in detail in this section, using two simplified models: a 1D shallowwater equations toy model and an adiabatic version of the ALADIN NWP model. This adiabatic version is interesting for two reasons: first, any effects due to the parameterizations of subgrid processes are filtered out, and second, the adiabatic version allows us to take larger 
time steps without rendering the numerical scheme unstable.

\section{a. Simplified models}

\section{1) SHALlow-WATER EQUATIONS TOY MODEL}

The shallow-water equations (SWE) are prototypical for the primitive equations based on the hydrostatic assumption, and are frequently used to test numerical schemes (Kalnay 2003; Termonia and Voitus 2008; Voitus et al 2009). The linearized one-dimensional SWE are given by

$$
\frac{D}{D t}\left(\begin{array}{c}
u \\
v \\
\phi
\end{array}\right)=\left[\begin{array}{ccc}
0 & f & -\frac{\partial}{\partial x} \\
-f & 0 & 0 \\
-c^{2} \frac{\partial}{\partial x} & 0 & 0
\end{array}\right]\left(\begin{array}{c}
u \\
v \\
\phi
\end{array}\right),
$$

or

$$
\frac{D}{D t} \mathbf{u}=\mathbf{L} \mathbf{u},
$$

where $D / D t=\partial / \partial t+U(\partial / \partial x), u$ and $v$ are the velocity perturbations, $\phi$ is the geopotential height, $U$ is the constant advection speed, $f$ is the Coriolis factor, and $c$ is the speed of the gravity waves.

These equations will be discretized and solved in exactly the same way as is done in the HARMONIE NWP system (i.e., with a spectral two-time-level semi-implicit semi-Lagrangian scheme; Haugen and Machenhauer 1993; Part I). One step of the time integration then consists of the following substeps:

1) Application of the explicit part of the operator $\mathbf{L}$ in spectral space:

$$
\mathbf{u}_{\exp }=\left(\mathbf{I}+\frac{\Delta t}{2} \mathbf{L}\right) \mathbf{u}^{t},
$$

where $\mathbf{u}^{t}$ is the solution at time $t$.

2) Inverse spectral transform of $\mathbf{u}_{\text {exp }}$.

3) Semi-Lagrangian interpolation:

$$
\mathbf{u}_{\mathrm{sl}}(x)=\mathbf{u}_{\exp }\left(x^{*}\right),
$$

where $x^{*}=x-U \Delta t$ is the departure point of a semiLagrangian trajectory. Cubic interpolation is used.

4) Coupling to the large-scale model: first, the largescale fields are periodized, either with the splinebased method (Part I ), or with the windowing-based method (Boyd 2005).

Then, the operator $\mathbf{I}-(\Delta t / 2) \mathbf{L}$ is applied to the periodized coupling fields $\mathbf{u}_{\mathrm{LS}}$, according to the method proposed in Radnóti (1995):
TABLE 1. Parameter values of the SWE model; $N$ is the total number of grid points.

\begin{tabular}{cl}
\hline \hline Parameter & Value \\
\hline$U$ & $50 \mathrm{~m} \mathrm{~s}^{-1}$ \\
$C$ & $300 \mathrm{~m} \mathrm{~s}^{-1}$ \\
$F$ & $10^{-4}$ \\
$\Delta x$ & $10 \mathrm{~km}$ \\
$N$ & 240 \\
$\Delta t$ & $400 \mathrm{~s}$ \\
\hline
\end{tabular}

$$
\tilde{\mathbf{u}}_{\mathrm{LS}}=\left(\mathbf{I}-\frac{\Delta t}{2} \mathbf{L}\right) \mathbf{u}_{\mathrm{LS}}^{t+\Delta t} .
$$

Finally, the LAM solution is coupled to these fields with Davies relaxation (Davies 1976):

$$
\tilde{\mathbf{u}}=\alpha \mathbf{u}_{\mathrm{sl}}+(1-\alpha) \tilde{\mathbf{u}}_{\mathrm{LS}},
$$

where $\alpha$ is the relaxation function.

5) Spectral transform of $\tilde{\mathbf{u}}$.

6) Solution of the implicit part of the operator $\mathbf{L}$ in spectral space finally yields the model state at the next time step:

$$
\mathbf{u}^{t+\Delta t}=\left(\mathbf{I}-\frac{\Delta t}{2} \mathbf{L}\right)^{-1} \tilde{\mathbf{u}} .
$$

The large-scale solution, to which the LAM solution is coupled, is obtained by integrating the SWE with the same numerical scheme at the same resolution, except that step 4 is replaced by the application of periodic boundary conditions (sufficiently far from the boundaries of the LAM model). By using the same numerical scheme, rather than an analytic solution, we guarantee that all differences between the large-scale solution and the LAM solution can be attributed to the coupling and periodization procedures.

Table 1 lists the parameter values used in the SWE toy model, which are representative for NWP applications. These will be used in all further experiments, except where stated explicitly. The initial condition in the toy model experiments will be a bell-shaped geopotential depression and geostrophic wind conditions $[u=0$ and $v=(1 / f)(\partial \phi / \partial x)]$.

\section{2) 3D MODEL IN ADIABATIC MODE}

As an intermediate step between the SWE toy model and a full 3D NWP model, we will consider the ALADIN NWP model in adiabatic mode. This means that all parameterizations of unresolved processes are removed, and only the solution of the hydrostatic 


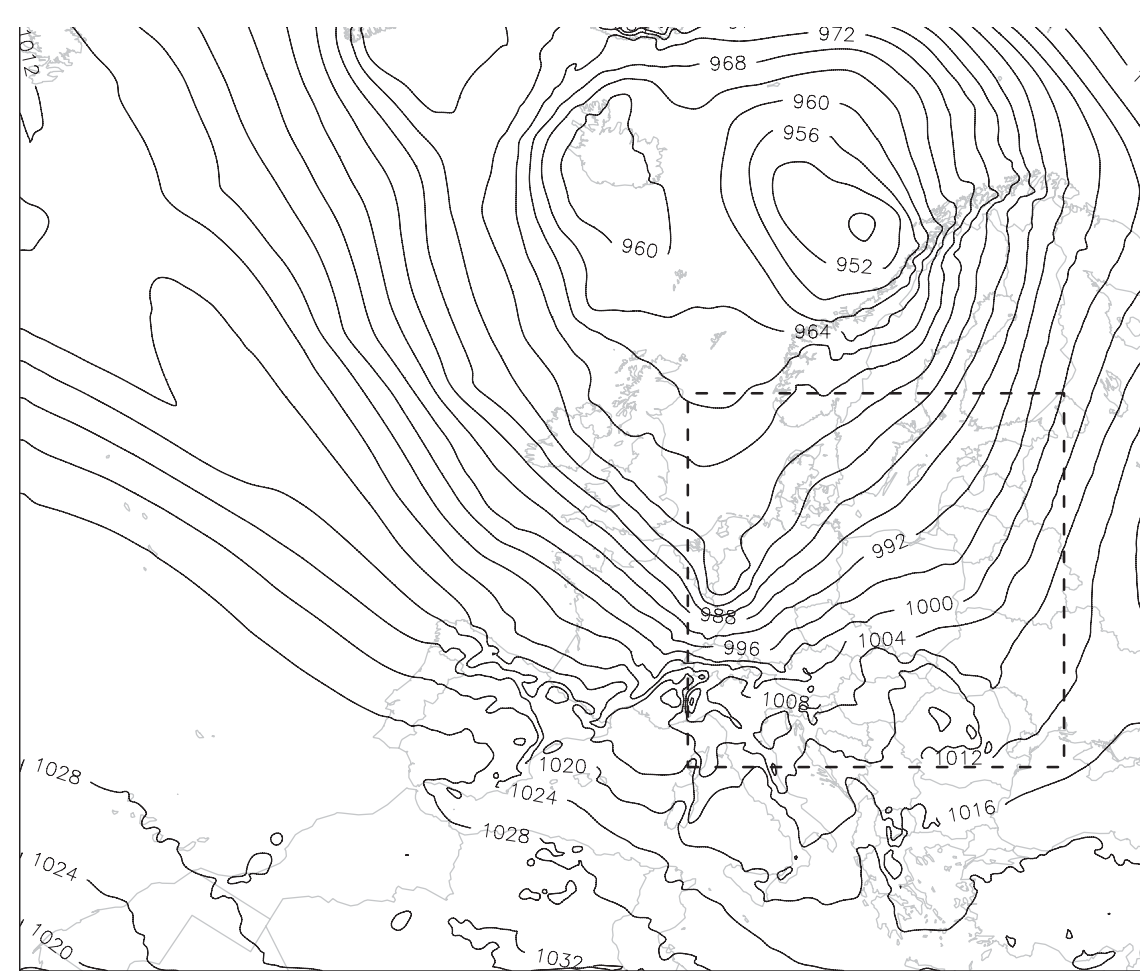

FIG. 1. MSLP from the adiabatic run on the host domain at the $10-\mathrm{h}$ forecast range. The dashed rectangle indicates the guest domain.

primitive equations remains. A detailed description of the numerical integration scheme employed by the ALADIN model is given in Part I. The spatial resolution is taken to be equal to $10 \mathrm{~km}$.

We will investigate the influence of relaxation and periodization with a perfect-model experiment setup (de Elía et al. 2002). This setup consists of two runs with the same model, at the same resolution, with the same time step, but on a different domain. The first run is done on a large "host" domain, and provides the coupling data for the second run on a smaller "guest" domain. Because both runs are performed with the same model, all differences can be attributed to the periodization and the coupling procedures. In this sense, the run on the host domain can be considered as the truth, which is why this approach is denoted a "perfect model" setup. This methodology will allow us to investigate accurately the difference between Boyd's proposal and the current procedure in the ALADIN NWP model, without using observations.

An important difference with the perfect model setup described by de Elía et al. (2002) is that they filter out the small scales from the coupling fields. This is done in order to mimic the operational situation where the coupling data usually originate from a lower-resolution (global) model, and to verify if the model can rebuild the small-scale features. To focus on the effects of the coupling and the periodization, we will not carry out this filtering in the experiments of this section. More operational-like perfect-model experiments are described in section 3 .

The experiments will be carried out for the famous and well-documented Lothar storm case of 26 December 1999. Wernli et al. (2002) give an in-depth description of the dynamical aspects of this case. The host domain is chosen large enough such that the complete cyclogenesis of the storm takes place inside the domain. The guest domain is taken such that the storm enters at full depth, at a forecast range of about $10 \mathrm{~h}$. All of the grid points of the guest domain coincide with grid points of the host domain in order to avoid interpolation errors. Figure 1 shows the mean sea level pressure (MSLP) at 10-h forecast range, along with the guest domain. This figure shows that the storm also develops when performing a run in adiabatic mode (i.e., when neglecting all parameterizations).

A possible problem with the modeling of such a fastmoving storm with a LAM model is the so-called temporal interpolation problem. It is common practice that the large-scale model provides coupling data only every $3 \mathrm{~h}$, and the fields are interpolated temporally at time steps in between. This strategy may largely degrade the 

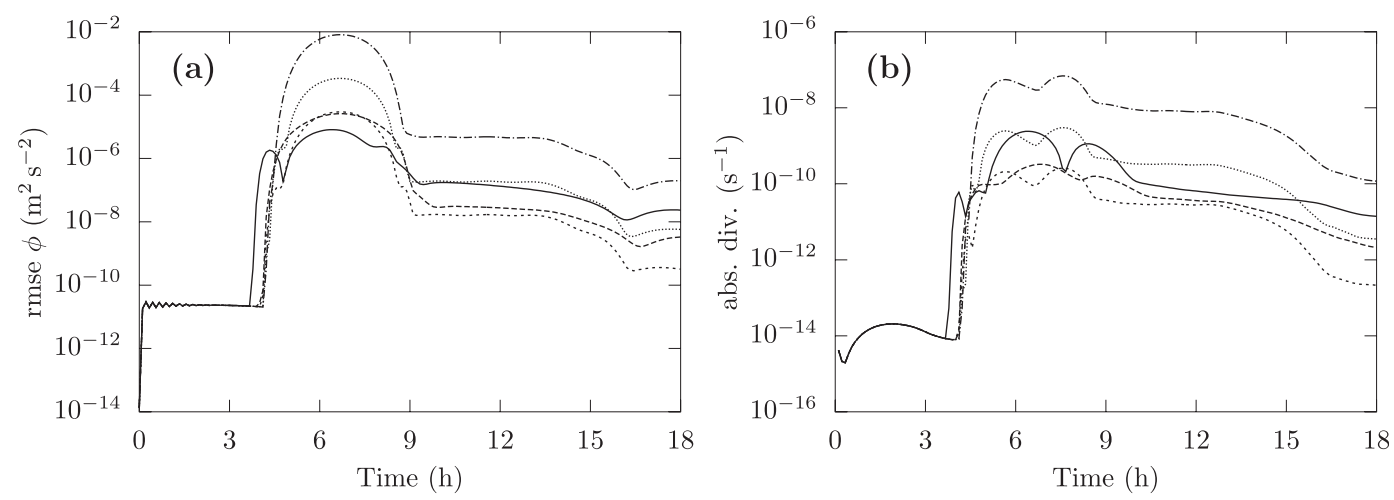

FIG. 2. Influence of the periodization method (Splines/Boyd) on (a) error on geopotential and (b) absolute divergence in SWE model with narrow extension zone (12 grid points). The configurations are SAST (solid), and BAST with $L=2$ (dashed), $L=3$ (short dashed), $L=5$ (dotted), and $L=8$ (dashed-dotted).

intensity of a storm entering the LAM domain (Termonia 2003; Tudor and Termonia 2010; Termonia et al. 2009). To avoid this problem, we will couple the guest model to the host model at every time step. In section 3 , the consequences of reducing the coupling update frequency will be examined.

\section{b. Periodization: Spline-based method and Boyd's method}

\section{1) SWE MODEL}

We will design this experiment carefully as to avoid any errors that are not due to the periodization. First, the initial state is a geostrophic depression, which is already inside the guest domain. By considering a signal that leaves the domain, instead of one that enters the domain, it is possible to eliminate errors due to the truncation of semi-Lagrangian trajectories at the boundary of the physical domain. Such errors are discussed later in section 2c. Second, as indicated in Table 1, the Courant number for our constant advection SWE model is an integer $(\mu=U \Delta t / \Delta x=2)$. This means that the semiLagrangian trajectories departure points coincide with grid points. As a consequence, no interpolation is needed, and no error is made in the treatment of the advection terms. Third, we choose a quite large relaxation zone of 48 points. Although this is not a realistic choice in an operational context, as it reduces the physical domain too strongly, it is useful here to reduce the impact of errors due to relaxation. Finally, the relaxation and periodization zones are considered not to overlap. This aspect will be discussed later in section $2 \mathrm{~d}$. In terms of the classification given above, the experiments in this section are SAST and BAST.

Boyd's periodization method has one tunable parameter, namely the scale parameter $L$. The experiments are carried out for several values for $L$. Figures 2 and 3 show the results for an extension zone of 12 and 48 points, respectively. Two error measures are considered: (i) the root mean squared error (rmse) of the geopotential $\phi$ (with respect to the host model, that is), and (ii) the absolute divergence, integrated over the domain. Since the initial state is geostrophic, the absolute divergence is a measure for the erroneous (gravity) waves generated by the periodization.

These figures show that the error is initially dominated by round-off errors, until the depression leaves the domain. Only at that moment, a signal is present at the border of the guest domain, and the periodization becomes relevant. For an extension zone of 12 grid points, the results with Boyd's method are only slightly better than with spline periodization. For a larger extension zone, however, the benefit of Boyd's method appears more clearly. For a well-chosen value of the scale parameter $L$, the error due to periodization even disappears completely. The optimal value for $L$ is around 2-3 for the narrow extension zone, and around 3-5 for the wide extension zone.

Comparing Figs. 2 and 3 also shows that the error with the 12-point extension zone is several orders of magnitude larger than with the 48-point extension zone. As already remarked in Boyd (2005), it should be noted that the width of the extension cannot be chosen freely, since larger extension zones imply computational overhead for the same physical domain. A trade-off has to be made between accuracy and efficiency when choosing this parameter.

\section{2) Adiabatic NWP MOdel}

We will now perform the same SAST and BAST experiments with the adiabatic NWP model for the Lothar storm case. The time step used in these experiments is equal to $450 \mathrm{~s}$. Since the initial fields of the guest model 

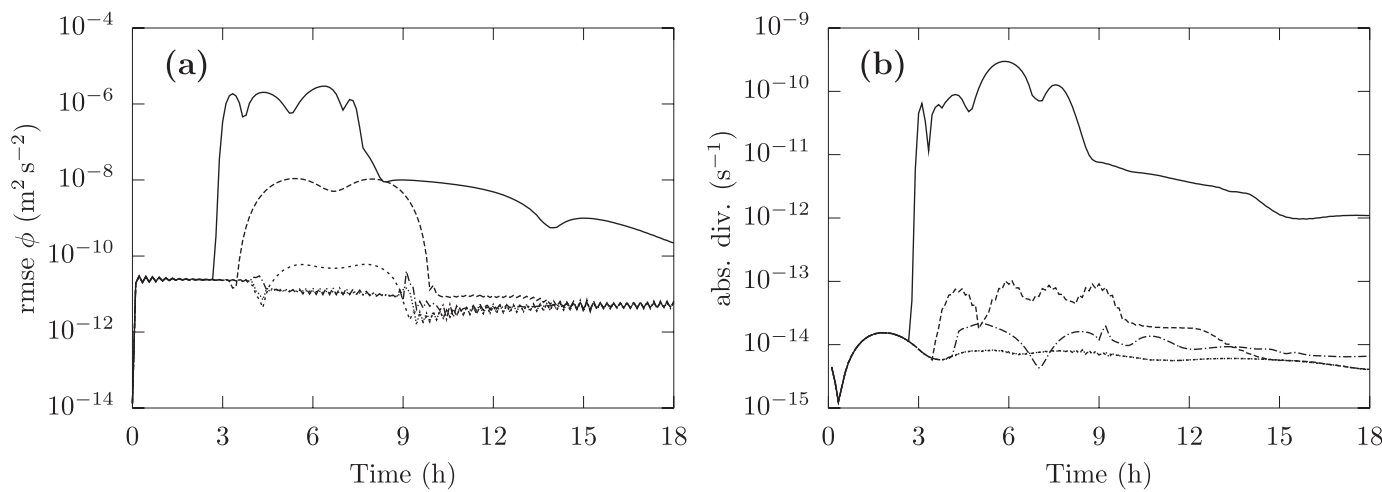

FIG. 3. Influence of the periodization method on (a) error on geopotential and (b) absolute divergence in SWE model with large extension zone (48 grid points). The configurations are SAST (solid); and BAST with $L=2$ (dashed), $L=3$ (short dashed), $L=5$ (dotted), and $L=8$ (dashed-dotted).

come directly from the host model, without interpolation, it is not necessary to perform a digital filter initialization.

Figures 4 and 5 show the rmse errors on the wind at $250 \mathrm{hPa}$ and on the MSLP, for a narrow (12 grid points) and a wide (48 grid points) extension zone. A first observation is that the results with Boyd's method are consistently better than with the spline-based method, for a wide range of the scale parameter $L$, both for a narrow and a wide extension zone.

A second observation is that, although Boyd's method improves the results, there is still a significant residual error, even for the wide extension zone. This is in contrast with the SWE model results, where the error completely vanished for an appropriate choice of the scale parameter $L$. This residual error can be attributed to procedures in the model that are beyond control, such as an elliptic spectral truncation to avoid aliasing, transformations between vorticity/divergence, and wind speeds, etc.
A third observation concerns the optimal value of the scale parameter $L$, which is different from the one obtained from the SWE model. In the 3D tests, larger values for $L$ yield better results, even for the narrow extension zone. As noted by Boyd, the optimal value for $L$ depends on the smoothness of the signal, which could explain this behavior.

\section{c. Truncation of SL trajectories}

The spline-based periodization method currently used in the HARMONIE system fills the extension zone with purely artificial content. To avoid such unphysical information entering the physical domain, the semi-Lagrangian trajectories are truncated at the border between the extension zone and the physical domain.

A key feature of Boyd's periodization method is that the signal is infinitely differentiable. As a consequence, the signal will be near-physical in a large region near the border between the extension zone and the physical
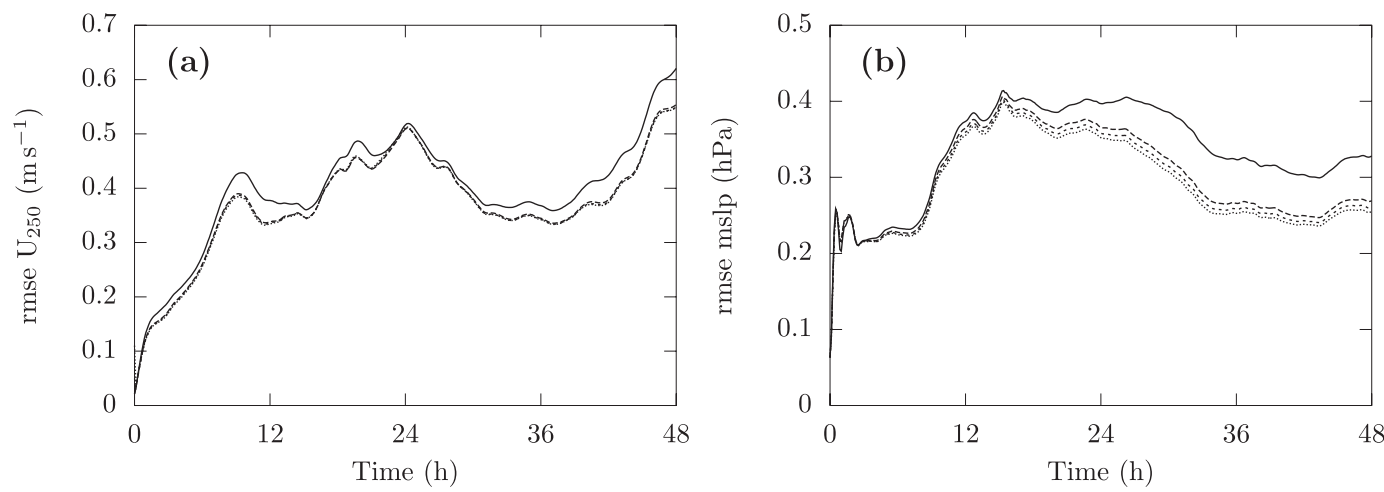

FIG. 4. Influence of the periodization method (Splines/Boyd) on (a) error on wind speed at $250 \mathrm{hPa}$ and (b) error on MSLP with an adiabatic model with narrow extension zone (12 grid points): SAST experiment (solid), and BAST experiments with $L=3$ (dashed), $L=5$ (short dashed), and $L=8$ (dotted). 

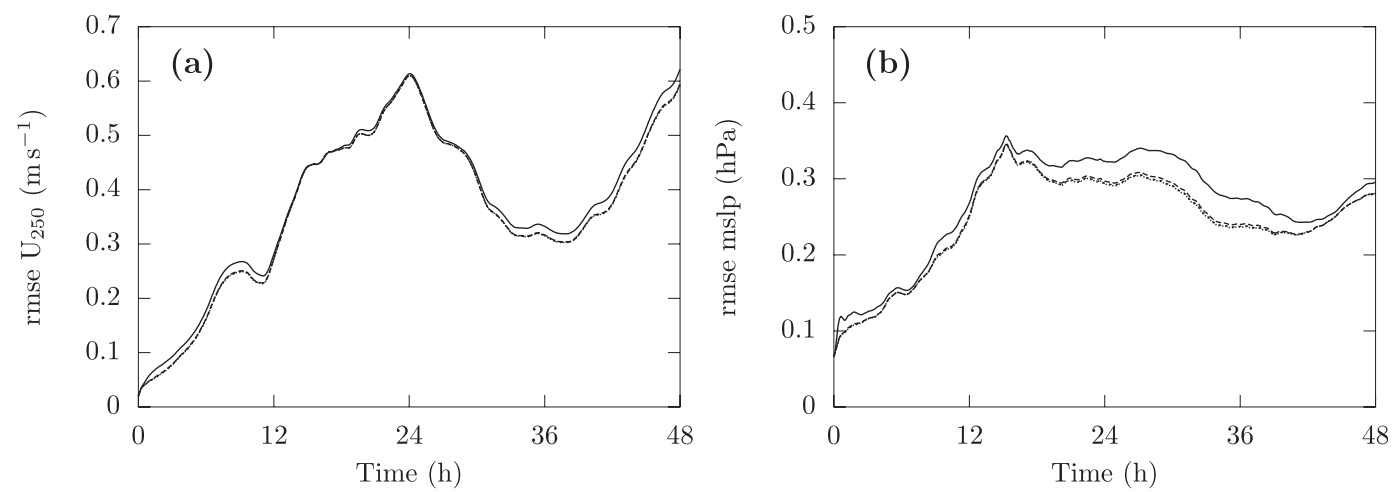

FIG. 5. Influence of the periodization method on (a) error on wind speed at $250 \mathrm{hPa}$ and (b) error on MSLP with adiabatic model with large extension zone (48 grid points): SAST experiment (solid), and BAST experiments with $L=3$ (dashed), $L=5$ (short dashed), and $L=8$ (dotted).

domain. This opens the question of whether it is still necessary to truncate the semi-Lagrangian trajectories when using Boyd's periodization method. Obviously, this decision also depends on the time step and the wind speed.

\section{1) SWE MODEL}

The experiment is again chosen such as to avoid any errors that are not due to the truncation of semiLagrangian trajectories. Therefore, a geostrophic initial state is considered, and a large extension zone (48 points) is chosen. As indicated before, it is possible to eliminate almost all errors due to the periodization with such an extension zone. The relaxation zone is taken large enough to minimize errors due to the coupling. The advection speed is kept at a value of $50 \mathrm{~m} \mathrm{~s}^{-1}$, but the time step is increased to $800 \mathrm{~s}$. This gives a Courant number of $\mu=4$, which is big but not exceptional for a semi-Lagrangian model. Since it is an integer number, no interpolation errors are made. For this experiment, we consider a depression that enters and leaves the domain.

Figure 6 shows the results for the spline-based method and Boyd's method, when truncating the semiLagrangian trajectories or not (i.e., for the SAST, BAST, SASN, and BASN configurations). This figure clearly illustrates why trajectory truncation is useful for the spline-based method: when the depression leaves the domain (at around $17 \mathrm{~h}$ ), the unphysical content of the extension zone reenters the domain at the other side, leading to an increase in the errors. For Boyd's method, on the other hand, this behavior does not occur, and both the entrance and the exit of the depression are described without significant error if the trajectories are not truncated.

\section{2) AdiABATIC NWP MODEL}

To highlight the effect of the truncation of the semiLagrangian trajectories, the time step of the adiabatic model was increased for these experiments to $900 \mathrm{~s}$.
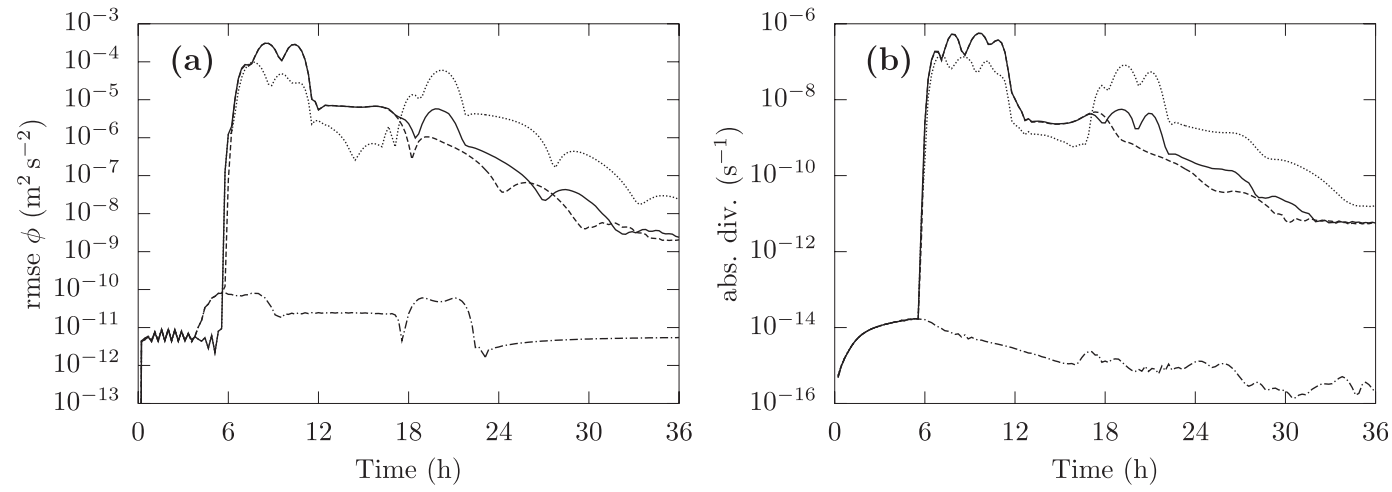

FIG. 6. Influence of truncation (Truncation/No truncation) of semi-Lagrangian trajectories on (a) error on geopotential and (b) absolute divergence in the SWE model: SAST (solid), BAST (dashed), SASN (dotted), and BASN (dashed-dotted). 

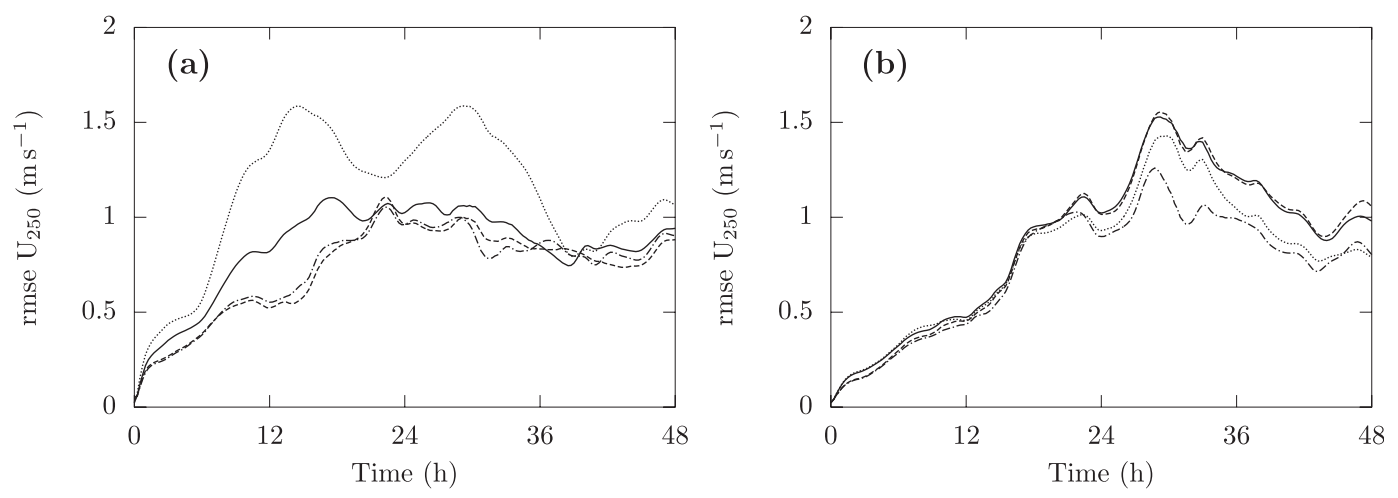

FIG. 7. Influence of truncation of semi-Lagrangian trajectories for adiabatic NWP model with (a) 12-point extension zone and (b) 48-point extension zone: SAST (solid), BAST (dashed), SASN (dotted), and BASN (dasheddotted). Note that these experiments were run with a time step of $900 \mathrm{~s}$.

Figure 7 shows the influence of the trajectory truncation with the spline-based periodization and Boyd's method, for an extension zone of 12 or 48 grid points. For 12 grid points, the trajectories extend relatively far into the extension zone, which means that unphysical information will enter the domain if the trajectories are not truncated. This is confirmed by the higher errors for the experiments without truncation. However, this effect remains relatively small when Boyd's periodization method is used, whereas the error is more severe in case of the spline-based method.

When considering a larger extension zone of 48 points, it turns out that trajectory truncation yields slightly larger errors. For such a wide extension zone, the first few grid points in the extension zone will contain near-physical information, which can be allowed to propagate into the domain. The figure shows that this is especially true when using Boyd's periodization method, as explained before.

\section{d. Relaxation with Boyd's method}

Boyd's proposal does not only include the periodization, but also the relaxation of the solution on the guest domain toward the solution on the host domain. In the implementation with the HARMONIE system the different configurations of the coupling can be used, see section $3 \mathrm{c}$ and Table 1 in Part I.

There are two options of the Davies relaxation function. The relaxation function used in the HARMONIE system is given by

$$
\alpha(x)=(p+1) x^{p}-p x^{p+1},
$$

which is defined on an interval $x \in(0,1)$, and where $p$ is a tunable parameter, which is assigned a value of 2.16 . Recall that we will denote an experiment with this function by a letter A. Boyd, on the other hand, proposes to use the same function as the bell window used in the periodization:

$$
\alpha(x)=\frac{1}{2}+\frac{1}{2} \operatorname{erf}\left(L_{r} \frac{2 x-1}{2 \sqrt{x-x^{2}}}\right),
$$

which is denoted by E. It is not strictly necessary to take the same value for the scale parameter $L_{r}$ for the relaxation as the one taken for the periodization. It is easy to show that for $L_{r}=1.36$, this function closely resembles the tuned HARMONIE relaxation function.

Regarding the location of the relaxation zone, the models in the HARMONIE system considers separate periodization and relaxation zones (denoted by $S$ ), whereas Boyd proposes to overlap the two zones (denoted by O).

\section{1) SWE MODEL}

For this experiment, a large extension zone (48 grid points) is considered, Boyd's periodization method is used with $L=3$, and the semi-Lagrangian trajectories are not truncated. Moreover, a geostrophic initial state is imposed and the parameters take the values specified in Table 1. As shown in the previous sections, these settings eliminate almost all errors that are due to the periodization, which will allow us to focus on the relaxation procedure. To emphasize the consequences of the relaxation, a smaller relaxation zone of 12 grid points is chosen.

Figure 8 shows the sensitivity of the error in the SWE model with respect to the choice of the relaxation function: the HARMONIE relaxation function (BASN experiment) and the erf-based relaxation function (BESN experiment) for $L_{r}=1.36, L_{r}=2$, and $L_{r}=3$. Apparently, the results are not very sensitive to the exact shape of the relaxation function, where the tuned HARMONIE function and the erf-based function 

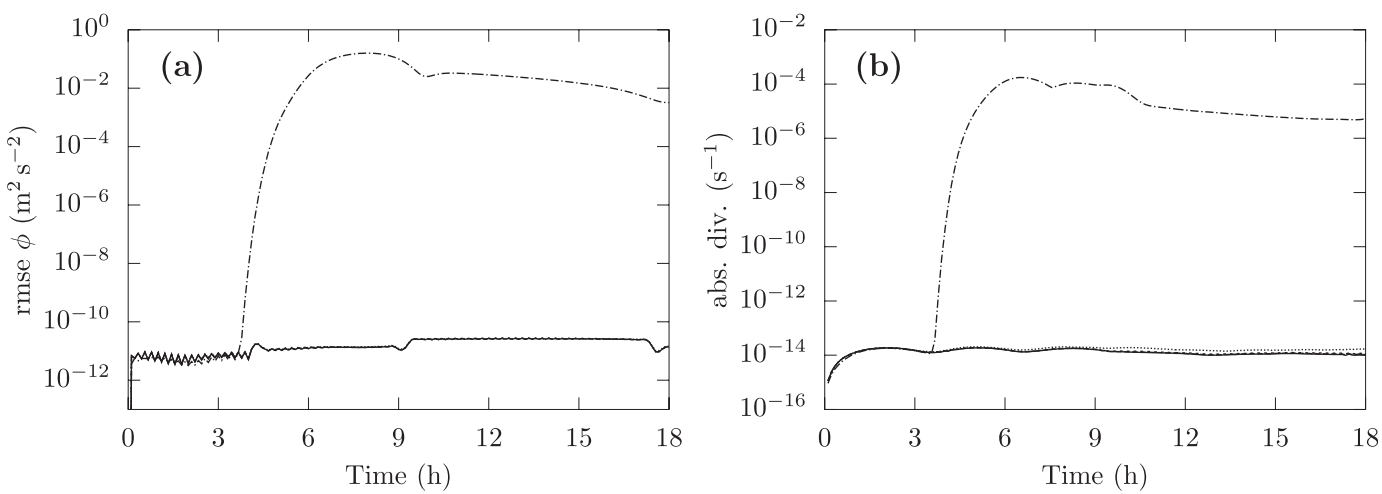

FIG. 8. Influence of the relaxation function (ALADIN/erf-based) and overlapping $(\mathrm{O})$ of relaxation and periodization zones on (a) error on geopotential and (b) absolute divergence in the SWE model: BASN (solid), BESN for $L_{r}=1.36$ (dashed), $L_{r}=2$ (short dashed), $L_{r}=3$ (dotted), and BAO (dashed-dotted).

with $L_{r}=1.36$ give the best results, while other values for $L_{r}$ yield slightly larger errors.

Figure 8 also shows that the error greatly increases when overlapping the relaxation and periodization zones (BAO experiment). As discussed in Part I, this can be attributed to the fact that Boyd considered an Eulerian framework, whereas we are working in a semiLagrangian framework, in which signals (and errors) can propagate much more easily from the relaxation zone into the physical domain. As a consequence, overlapping does not seem advisable for semi-Lagrangian models.

\section{2) Adiabatic NWP MOdel}

Figure 9 shows the influence of the relaxation function on the error in the adiabatic NWP model. For all these experiments, Boyd's periodization method was used on an extension zone of 12 grid points. The results are quite consistent with the behavior of the SWE model. The error is not very sensitive to the exact shape of the relaxation function, with the tuned relaxation function in HARMONIE (BASN) giving the best results, and the erf-based relaxation function with $L_{r}=1.36$ giving only slightly worse results (BESN). The influence of overlapping the relaxation and the extension zones is more important, with overlapping yielding significantly larger errors (BAO). The time evolution of the MSLP error indicates that the initial fields are no longer well balanced.

Moreover, the gain in computational efficiency due to overlapping is rather limited. The most expensive part of a NWP model are the physics parameterizations. The Radnóti coupling strategy (Radnóti 1995) used in the HARMONIE NWP system allows to avoid these calculations in the extension zone, because its content is completely determined by the solution from the host model. When overlapping the relaxation zone and the extension zone, this is no longer true, and physics calculations in the extension zone become necessary.
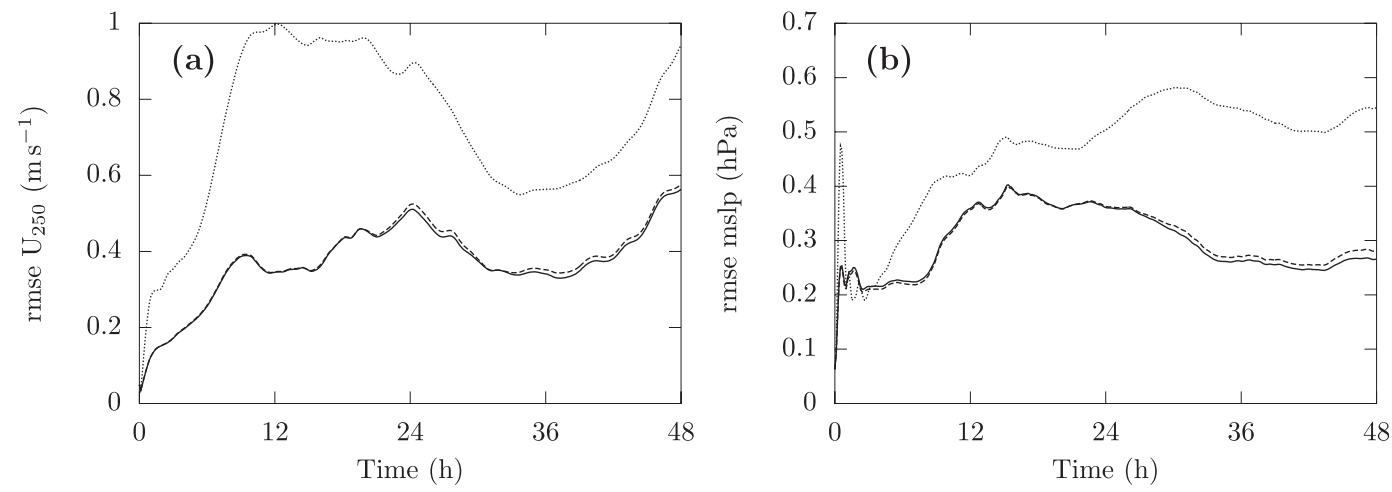

FIG. 9. Influence of relaxation function and overlapping of coupling and relaxation zones on (a) wind speed at $250 \mathrm{hPa}$ and (b) MSLP from the adiabatic NWP model: BASN (solid), BESN for $L_{r}=1.36$ (dashed), and BAO (dotted). 

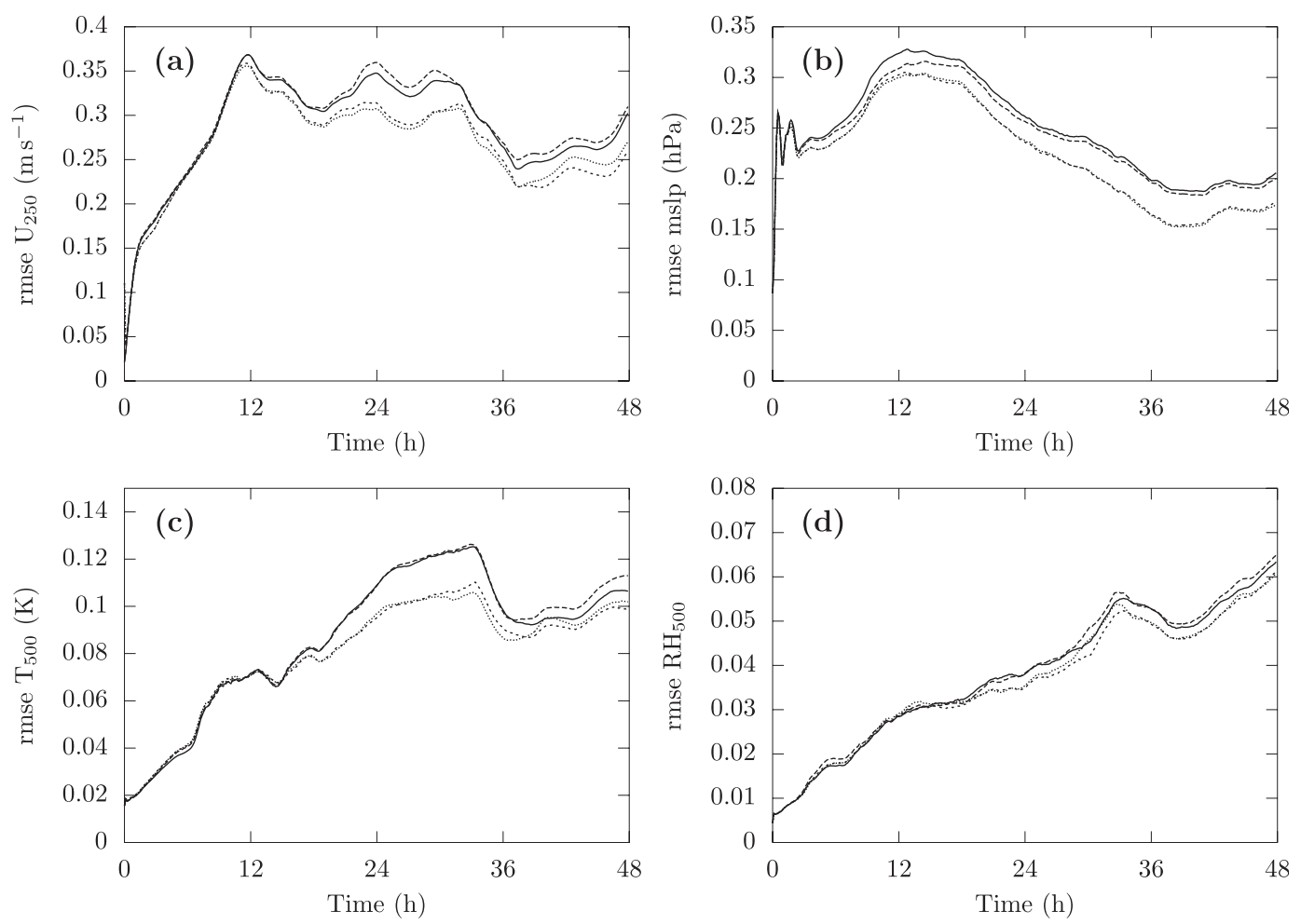

FIG. 10. Influence of periodization (Boyd/Splines) and truncation (Truncation/No truncation) of semi-Lagrangian trajectories on NWP model run with activated physics parameterizations: SEST (solid), SESN (dashed), BEST (short dashed), and BESN (dotted). (a) 250-hPa wind, (b) MSLP, (c) 500-hPa temperature, and (d) 500-hPa relative humidity.

\section{Impact of Boyd's method under operational conditions}

Until now, several assumptions and tricks were used to idealize the experiments. First, an adiabatic NWP model was used, with the parameterization of subgrid phenomena disabled. Second, the host model providing the coupling data was run at the same resolution as the guest model. Third, the host model provided coupling data every time step, instead of the more usual every $3 \mathrm{~h}$. These idealizations raise the question whether the conclusions of the previous section still stand when moving to operational conditions.

In this section we will go stepwise toward more operational-like experiments. The parameters studied in the previous section will be given constant values: the scale parameter $L$ is given a value of 8 , which was found to be optimal for our domain. As discussed earlier, the results are not very sensitive to the choice of the relaxation function. For the following experiments the erfbased relaxation function is used with a scale parameter $L_{r}=1.36$. The extension zone is chosen to have a width of 12 grid points, which is a reasonable trade-off between computational cost and accuracy. The time step for all experiments is taken to be $450 \mathrm{~s}$. The relaxation zone and extension zone are not overlapped. The model will be run in the following four configurations: SEST, BEST, SESN, and BESN. This will allow us to determine the influence of the periodization (S or B) and the influence of the truncation of the semi-Lagrangian trajectories ( $\mathrm{T}$ or $\mathrm{N}$ ).

\section{a. Activating the physical parameterizations}

For the experiments in this section, we use the ALARO physics parameterizations described in Gerard et al. (2009), which is available in the HARMONIE system. The run on the guest domain is still coupled every time step to a run on the host domain at the same resolution.

Figure 10 shows the rmse for some relevant fields: the 250-hPa wind, the mean sea level pressure, the 500-hPa temperature, and the 500-hPa relative humidity. The different figures show results similar to what was found in the adiabatic tests: experiments based on Boyd's method give consistently better results than the experiments with spline-periodized fields. Including the physics does not conceal the advantage of Boyd's periodization method. 


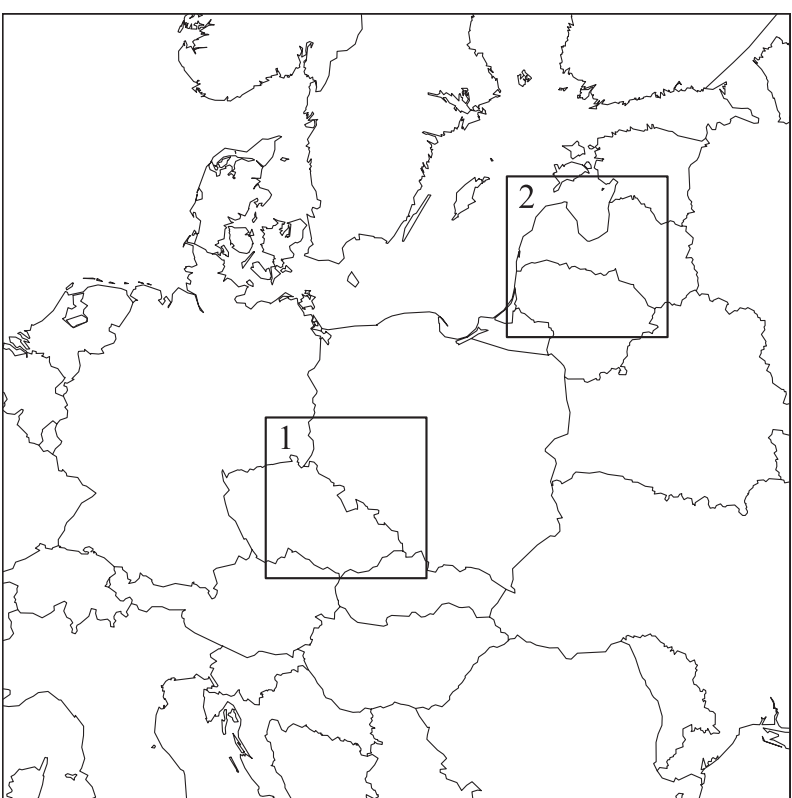

FIG. 11. Definition of the subdomains.

This conclusion equally holds for parameterizationrelated fields such as the relative humidity.

It is interesting to have a closer look at the time evolution of the errors at different locations in the domain. To this goal, we consider the two subdomains shown in Fig. 11. Both domains are lying close to the track of the center of the storm. The errors for the MSLP and the $250-\mathrm{hPa}$ zonal wind are presented for the two domains in Figs. 12 and 13, respectively.

In general, Boyd's method gives the smallest errors. Shortly before the arrival of the storm in the subdomain 2 , however, the rmse with the spline periodization is a bit lower. This difference is, however, negligible compared with the differences found later in the forecast. The peaks in the rmse correspond with the passage of the storm in the subdomains. After the arrival of the storm depression in the second subdomain, we recognize a large improvement of Boyd's method compared to splines.

\section{b. Coupling to a lower-resolution host model}

The original perfect-model approach proposed by de Elía et al. (2002) consists of (i) a run on a high-resolution host domain, (ii) interpolation to a low-resolution host domain, and (iii) using these data as coupling data for a run on the high-resolution guest domain. As explained in section 2, the intermediate interpolation was avoided in the previous experiments in order to focus on the periodization and the relaxation effects. In this section, the intermediate interpolation is introduced in order to better mimic an operational situation. The low-resolution host domain has a resolution of $40 \mathrm{~km}$.

Figure 14 presents the results for the $250-\mathrm{hPa}$ wind and the mean sea level pressure. A general increase of the error is observed in comparison to the previous experiments, due to the loss of some small-scale information. Boyd's method still gives better results than the spline method.

\section{c. Reducing the coupling update frequency}

In the experiments before, the host model provided the guest model with coupling data every time step. In an operational context this is infeasible, and a common choice for the updating the coupling data is every $3 \mathrm{~h}$. At time steps in between, the coupling data are interpolated in time.

As shown by Termonia (2003), reducing the coupling update frequency may generate significant errors in case of a fast-moving storm such as the Lothar storm. Nevertheless, we will investigate the impact of increasing the coupling update interval from one time step (450 s) to 2 , 4,8 , and 24 time steps $(15 \mathrm{~min}, 30 \mathrm{~min}, 1 \mathrm{~h}$, and $3 \mathrm{~h}$, respectively).
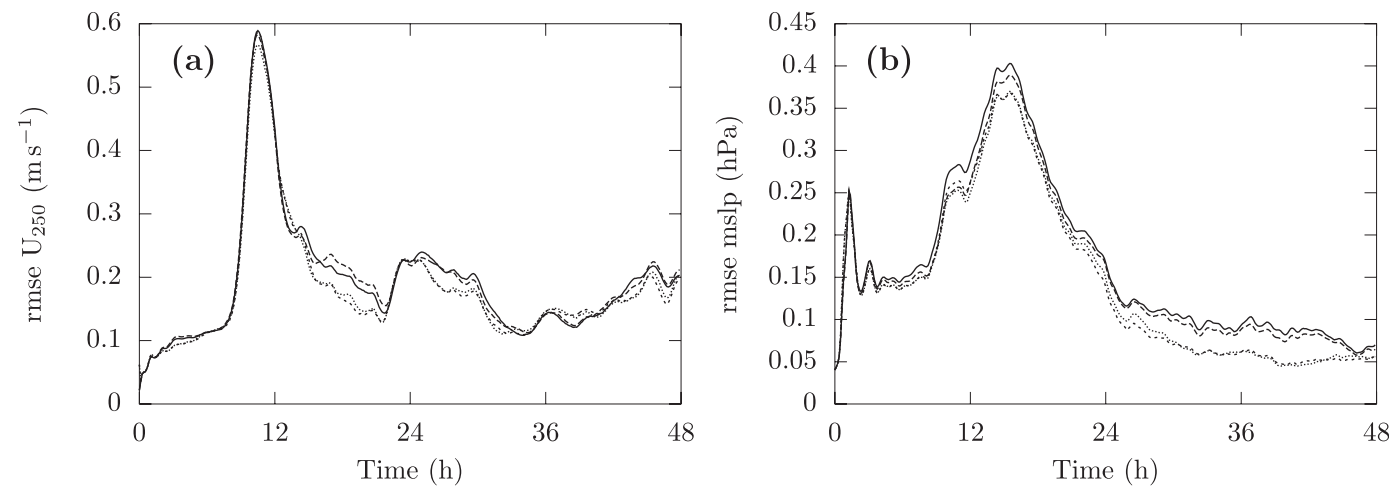

FIG. 12. Error on (a) 250-hPa wind and (b) MSLP in subdomain 1, where the storm arrives after about 12 h: SEST (solid), SESN (dashed), BEST (short dashed), and BESN (dotted). 

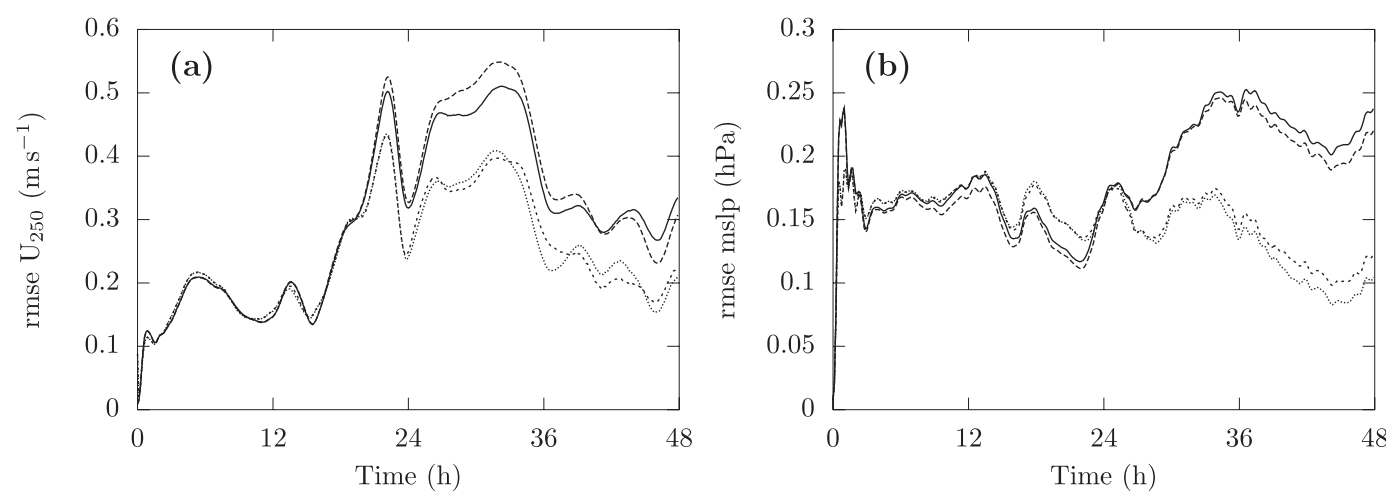

FIG. 13. Error on (a) 250-hPa wind and (b) MSLP in subdomain 2, where the storm arrives after about 20 h: SEST (solid), SESN (dashed), BEST (short dashed), and BESN (dotted).

Figure 15 shows the results for these different intervals. Again, Boyd's method gives better results than the spline method, but the relative difference is decreasing because the absolute errors increase with larger coupling intervals. From these results it can be recommended that, for this case of a fast-moving storm, the coupling-update frequency should be less than or equal to $1 \mathrm{~h}$. For a coupling update interval of $3 \mathrm{~h}$, the error is entirely dominated by the time interpolation problem (Termonia 2003), and the advantage of Boyd's method is concealed. Note that applying the error originating from the temporal interpolation can be monitored in an operational application, see Termonia (2004), which could in principle allow us to increase the update frequency temporarily in such cases.

\section{Conclusions}

We performed extensive tests to evaluate Boyd's proposal for the periodization and relaxation of the coupling fields in a spectral LAM. Starting with idealized models (a 1D SWE toy model of a depression and a 3D adiabatic NWP model for the Lothar storm case) we studied different aspects of Boyd's proposal in a perfect model setup. Regarding the periodization, our tests show that Boyd's method gives consistently better results than the spline-based method currently used in the HARMONIE NWP system. Boyd's method is also more tolerant with respect to semi-Lagrangian trajectories leaving the physical domain. Regarding the relaxation, no significant difference was found between the erf-based relaxation function proposed by Boyd and the tuned function currently used in the HARMONIE NWP system. For our semi-implicit semiLagrangian model, however, one aspect of Boyd's proposal appears detrimental for the accuracy, namely, the overlapping of the relaxation zone and the extension zone.

To mimic operational NWP conditions, experiments were performed in which the physics parameterizations were activated and the coupling data were provided at a lower resolution and at a lower rate. These experiments
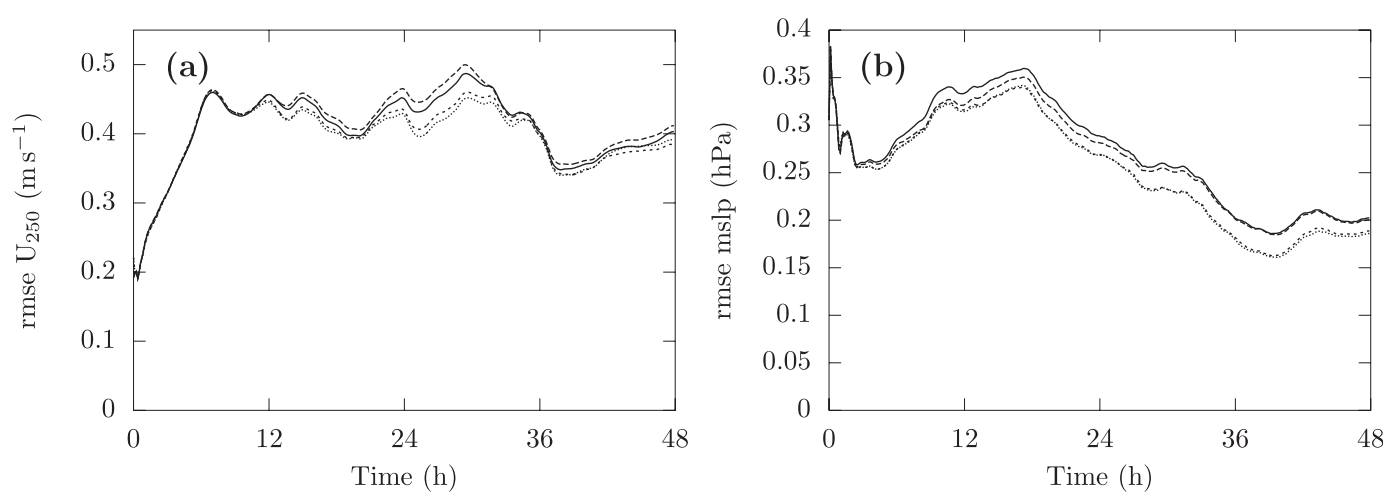

FIG. 14. Influence of periodization method (Splines/Boyd) and truncation (Truncation/No truncation) of semiLagrangian trajectories on (a) error on 250-hPa wind and (b) error on MSLP when coupling to low-resolution (40 km) data: SEST (solid), SESN (dashed), BEST (short dashed), and BESN (dotted). 

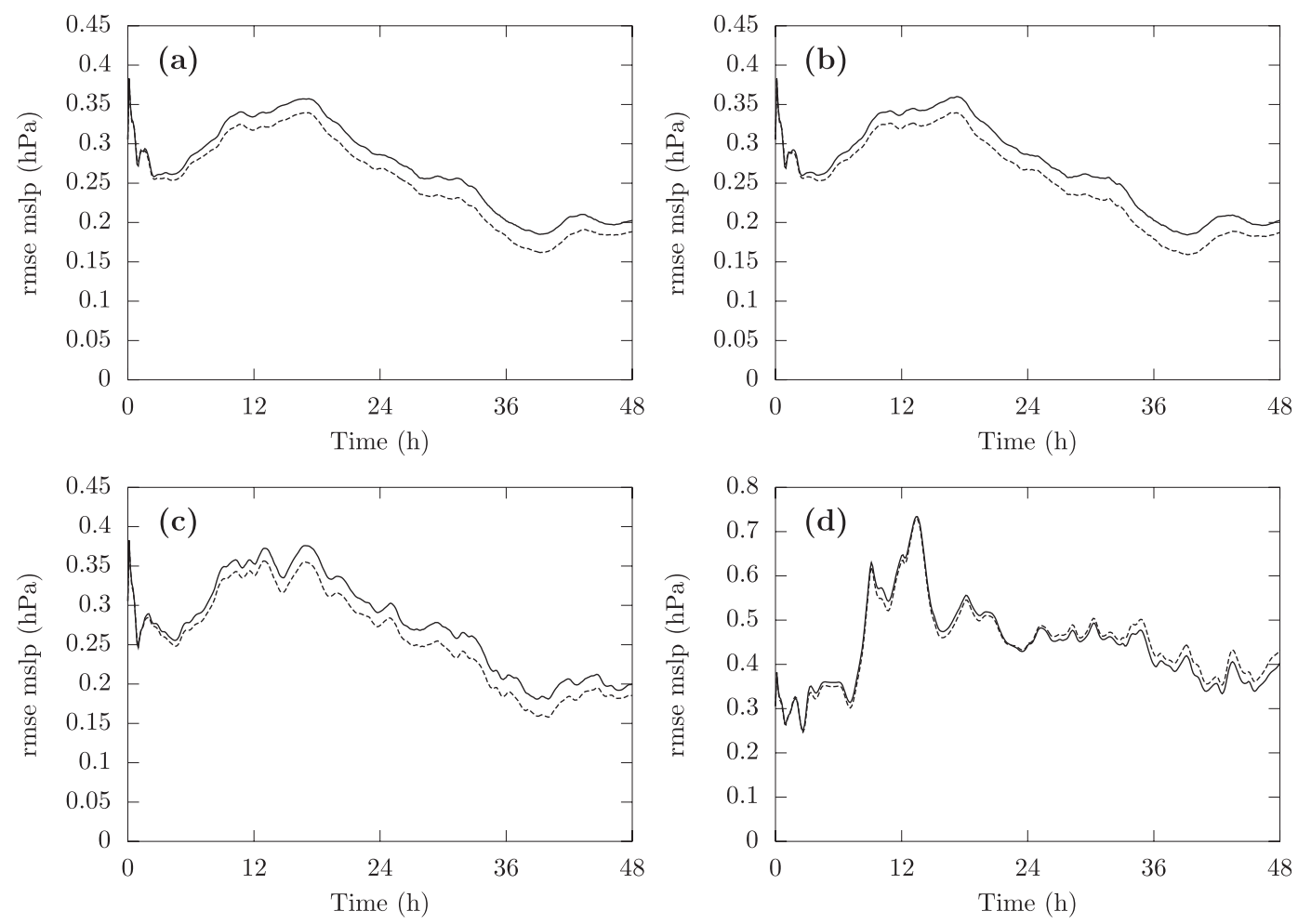

FIG. 15. Influence of periodization method (Splines/Boyd) on error on MSLP for different coupling update intervals: (a) $15 \mathrm{~min}$, (b) $30 \mathrm{~min}$, (c) $1 \mathrm{~h}$, and (d) $3 \mathrm{~h}$ : SEST (solid) and BEST (dashed).

show that the conclusions from the idealized experiments remain valid in an operational context, and allow us to deduce the following specific recommendations for periodization and relaxation within the HARMONIE system:

- Boyd's windowing method outperforms the splinebased periodization method;

- the current relaxation function and Boyd's erf-based relaxation function perform quite similarly;

- the relaxation and periodization zones should not overlap;

- truncation of semi-Lagrangian trajectories is not strictly necessary with Boyd's windowing method.

In short, the recommended configuration is BASN or BESN.

These results clearly indicate the superiority in terms of accuracy of Boyd's windowing-based method with respect to the spline method within a Fourier spectral model. Although Part I found no significant improvement of the scores over a longer validation period, the results of the present paper demonstrate improvements in singular storm cases that are highly relevant for meteorological applications. Even if, in practice, these improvements may be concealed by the use of too long a coupling-update frequency or by the model errors originating from the physics parameterizations, one may keep in mind, as J.P. Boyd put it himself, that "there is no reason to do badly what can be done well." This is particularly important in models that are under permanent development aimed at decreasing the other model errors. For instance, if, at some time in the future, one decides to run the LAM with a coupling-update time of $1 \mathrm{~h}$ instead of $3 \mathrm{~h}$, the benefits will not be hampered by the inaccuracy of the spline method.

Acknowledgments. This work was supported by the Belgian Science Policy unit, Research Project MO/34/ 021. The authors also thank the two anonymous reviewers for their valuable comments.

\section{REFERENCES}

ALADIN International Team, 1997: The ALADIN project: Mesoscale modelling seen as a basic tool for weather forecasting and atmospheric research. WMO Bull., 46, 317-324.

Boyd, J. P., 2005: Limited-area Fourier spectral models and data analysis schemes: Windows, Fourier extension, Davies relaxation, and all that. Mon. Wea. Rev., 133, 2030-2042.

Davies, H. C., 1976: A lateral boundary formulation for multilevel prediction models. Quart. J. Roy. Meteor. Soc., 102, 405-418. 
de Elía, R., R. Laprise, and B. Denis, 2002: Forecasting skill limits of nested, limited-area models: A perfect-model approach. Mon. Wea. Rev., 130, 2006-2023.

Gerard, L., J.-M. Piriou, R. Brožková, J.-F. Geleyn, and D. Banciu, 2009: Cloud and precipitation parameterization in a mesogamma-scale operational weather prediction model. Mon. Wea. Rev., 137, 3960-3977.

Haugen, J. E., and B. Machenhauer, 1993: A spectral limited-area model formulation with time-dependent boundary conditions applied to the shallow-water equations. Mon. Wea. Rev., 121, 2618-2630.

Kalnay, E., 2003: Atmospheric Modeling, Data Assimilation and Predictability. Cambridge University Press, 364 pp.

Radnóti, G., 1995: Comments on "A spectral limited-area formulation with time-dependent boundary conditions applied to the shallow-water equations." Mon. Wea. Rev., 123, 3122-3123.

Termonia, P., 2003: Monitoring and improving the temporal interpolation of lateral-boundary coupling data for limited-area models. Mon. Wea. Rev., 131, 2450-2463.

- 2004: Monitoring the coupling-update frequency of a limitedarea model by means of a recursive digital filter. Mon. Wea. Rev., 132, 2130-2141.
_ - and F. Voitus, 2008: Externalizing the lateral boundary conditions from the dynamic core in semi-implicit semiLagrangian models. Tellus, 60A, 632-648.

—- A. Deckmyn, and R. Hamdi, 2009: Study of the lateral boundary condition temporal resolution problem and a proposed solution by means of boundary error restarts. Mon. Wea. Rev., 137, 3551-3566.

, F. Voitus, D. Degrauwe, S. Caluwaerts, and R. Hamdi, 2012: Application of Boyd's periodization and relaxation method in a spectral atmospheric limited-area model. Part I: Implementation and reproducibility tests. Mon. Wea. Rev., 140, 3137-3148.

Tudor, M., and P. Termonia, 2010: Alternative formulations for incorporating lateral boundary data into limited-area models. Mon. Wea. Rev., 138, 2867-2882.

Voitus, F., P. Termonia, and P. Bénard, 2009: Well-posed lateral boundary conditions for spectral semi-implicit semiLagrangian schemes: Tests in a one-dimensional model. Mon. Wea. Rev., 137, 315-330.

Wernli, H., S. Dirren, M. A. Liniger, and M. Zillig, 2002: Dynamical aspects of the life cycle of the winter storm 'Lothar' (24-26 December 1999). Quart. J. Roy. Meteor. Soc., 128, 405-429. 